\title{
Design and Experiment Research of Micro-feed Self-sensing Micro-Driving System Based on GMM
}

\author{
Zhen Yu, Jie Mei, and Ding-fang Chen, Member, IACSIT
}

\begin{abstract}
The micro-driving system driven by Giant Magnetostrictive Material (abbr. GMM) has a lot of advantages such as large feed force, high displacement resolution, wide displacement range and so on, which overcome the shortcoming of the PZT driver such as its intricate structure to prevent leakage since the PZT must work in high-voltage situation. When the magnetic field changed, submicron displacement output is realized by the micro-driving device, with the function of self-sensing micro-feed of the micro-driving system, it is easy to realize automatic compensation of the micro-feed, so the application prospect of the micro-driving system driven by GMM used in the field of Super-precision manufacturing is extensive.
\end{abstract}

Index Terms-Giant magnetostrictive material; micro-driving system; micro-feed self-sensing; labview; precision strain gauge

\section{INTRODUCTION}

Magnetostrictive phenomenon means that ferromagnetic crystal can generate deformation when it's in magnetic field, and its magnetostrictive deformation can be used in fields of micro-feed[1]. Giant Magnetostrictive Material (abbr. GMM) can turn the magnetic energy into mechanical energy[2], which has been used in the field of fluid control components[3], micro driving valve[4], transducer[5], etc., GMM is regarded as a kind of strategic functional material to improve a country's high-tech integrated competitiveness in 21 century.

The advantages of the GMM such as large extension rate, high magnetism-mechanism coupling property and quick response, makes the micro-driving system based on GMM be characteristics of [6,7]micro-feed, high accurate response, strong driving ability and quick response, etc. The prospects of the system applied in super-precision manufacturing field and other micro-feed fields will be very comprehensive.

\section{MICRO-DRIVING PRINCIPLE OF GMM}

Magnetostrictive metamorphose is related with the process of magnetization[7], including spontaneous magnetization metamorphose and initiative magnetization metamorphose. The spontaneous magnetization metamorphose process is shown in fig. 1 , where the dotted circle is the original shape, when the temperature is lower

Manuscript received June 22, 2011, revised September 30, 2011. Zhen YU is with the College of Machinery and Automation, WuHan University of Science and Technology, WuHan, China (e-mail:for chenyu@ sohu.com).

Jie MEI and Ding-fang CHEN are with Intelligent Manufacturing \& Controlling Institute, WuHan University of Technology,WuHan, China (e-mail: jiemeiben@hotmail.com; cadcs@126.com). than the Courier temperature, the spontaneous magnetization metamorphose happens. The magnetic domain is elongated along a direction and shortened along the other direction; as a result, the globoid of single magnetic domain is turned into ellipsoid (noted by the solid line figure in fig. 1 ).

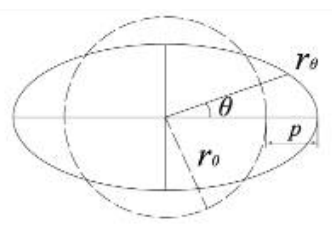

Fig.1

Fig.1. Spontaneous magnetization metamorphose of single magnetic domain

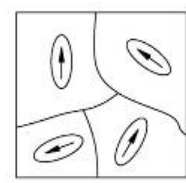

Fig.2(a)

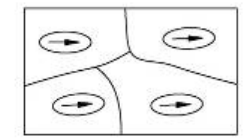

Fig.2(b)
Fig.2. Magnetization Metamorphose of GMM((a) Spontaneous Magnetization Metamorphose,(b) Initiative Magnetization Metamorphose)

Because of the anisotropy of GMM, the magnetization direction of the magnetic domain is unsystematic in the course of spontaneous magnetization, so the radius of the ellipsoid is different along different magnetization direction. Suppose the average radius of the GMM in macro state is $\bar{r}$, we have the following formula:

$$
\begin{aligned}
& \bar{r}=\int_{0}^{2 \pi} \int_{0}^{\pi} r_{\theta} \sin \theta d \theta d \varphi / 4 \pi \\
& =\int_{0}^{2 \pi} \int_{0}^{\pi}\left(r_{0}+p\left(3 \cos ^{2} \theta-1\right) / 2\right) \sin \theta d \theta d \varphi / 4 \pi \\
& =r_{0}+\frac{p}{2}\left(3 \times \frac{1}{3}-1\right)=r_{0}
\end{aligned}
$$

where $r_{0}$ is the radius of the globoid before spontaneous magnetization metamorphose, $r_{\theta}$ is the radius of any point of the ellipsoid after spontaneous magnetization metamorphose, $\theta$ is the angle from the point to long axis of the ellipsoid, and $p$ is the largest metamorphose.

Formula (1) shows the average radius of the ellipsoid along all direction is equal to $r_{0}$, so no metamorphose is happened in macro state when the GMM is in the state of spontaneous magnetization (shown in fig.2 (a)).

The initiative magnetization metamorphose process of the GMM is shown on fig.2 (b). If the magnetic field is strong enough to make the magnetic torque of the magnetic domain go along the same direction, all long axis of the ellipsoid turn into the direction along the outer magnetic 
field, so that the GMM is lengthened along the direction, while being shortened along the orthogonal direction. The process is called metamorphose of GMM, due to magnetic field, according to the change of magnetization intensity, that is how the magnetostrictive metamorphose happened.

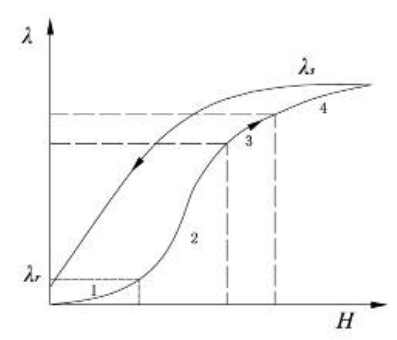

Fig. 3. The magnetostrictive process of GMM

In fig. $3 H$ is outer magnetic intensity, $\lambda$ is magnetostrictive extension rate, $\lambda_{\mathrm{s}}$ is saturated magnetostrictive extension rate, $\lambda_{r}$ is remanent magnetostrictive extension rate. In fig. 3 , the magnetostrictive trait of the GMM is divided into four phases, which is reversible magnetostrictive process, irreversible magnetostrictive process, the rotational process of the magnetic torque of the magnetic domain and the saturated process of the magnetization (shown in curve $1,2,3,4$ in fig. 3 ). The micro-driving principle of the GMM is caused by the irreversible magnetostrictive process.

\section{DESIGN OF MICRO-DRIVING DEVICE BASED ON GMM}

\section{A. Size Calculation of GMM Stick for Micro-Driving Device}

The core part of the micro-driving device is the GMM stick, whose geometrical parameter includes length $l_{\text {stick }}$ and diameter $d_{\text {stick }} \cdot l_{\text {stick }}$ is calculated according to the micro-feed of the micro-driving device, $d_{\text {stick }}$ is decided according to its output force. In general, the longest protraction of the GMM stick is equal to the largest micro-feed of the micro-driving device $(\Delta l)$, so $l$ is calculated by (2):

$\Delta l=\frac{l_{\text {stick }} \times \lambda}{k} \Rightarrow l_{\text {stick }}=k \frac{\Delta l}{\lambda}$

where $\Delta l$ is the longest protraction of the GMM stick, $\lambda$ is Magnetostrictive rate $\left(\lambda=900 \times 10^{-6}\right)$, and $k$ is a coefficient (equals to 1.2 ). According to manufacturers' standards and its driving force demand, the geometrical parameter of the GMM stick is: $l_{\text {stick }}=50 \mathrm{~mm}$, $d_{\text {stick }}=8 \mathrm{~mm}$, and the largest output displacement of the micro-driving device is $66.6 \mu \mathrm{m}$ calculated by (2).

\section{B. The Driving Magnetic Field Intensity Made on the GMM Stick}

Given the driving magnetic field intensity for the GMM stick is $H_{q}, \lambda_{m}=900 \times 10^{-6}$, according to fig.4[5], $H_{q}$ is within the scope of $0 \sim 50 \mathrm{kA} / \mathrm{m}$.

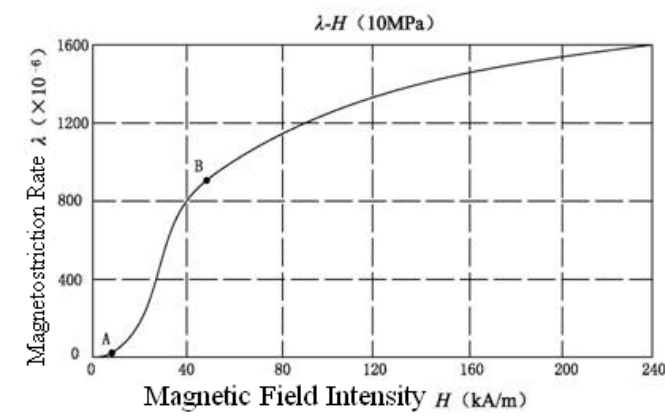

Fig. 4. Relationship between $\lambda$ and $H$ of the GMM

The driving magnetic field of the micro-driving system is produced by a solenoid coil. The parameters of the solenoid coil include $r_{1}, r_{2}, l$ (see fig.5)and $d$ ( the copper wire diameter of the solenoid), thus the central magnetic field intensity distribution of the solenoid is[8]:

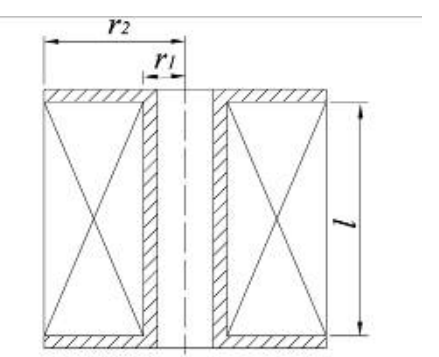

Fig. 5. Schematic diagram of solenoid coil

$$
H_{x}=2 \pi n_{1} n_{2} I\left\{\begin{array}{l}
(x+l / 2) \ln \frac{r_{2}+\left[r_{2}^{2}+(x+l / 2)^{2}\right]^{1 / 2}}{r_{1}+\left[r_{1}^{2}+(x+l / 2)^{2}\right]^{1 / 2}} \\
+(l / 2-x) \ln \frac{r_{2}+\left[r_{2}^{2}+(l / 2-x)^{2}\right]^{1 / 2}}{r_{1}+\left[r_{1}^{2}+(l / 2-x)^{2}\right]^{1 / 2}}
\end{array}\right\}
$$

In (3), $n_{1}$ and $n_{2}$ are coil turns per unit length and unit height of the solenoid respectively, and $H_{x}$ is magnetic field intensity of $x$ point from the center of solenoid. According to the design: $d$ equals to $0.8 \mathrm{~mm}$, the coil turns is 1500 , $l$ equals to $0.07 \mathrm{~m}, r_{1}$ equals to $0.022 \mathrm{~m}$, and $r_{2}$ equals to $0.04 \mathrm{~m}$. When $X$ equals to $0, H_{0}$ (the center magnetic field intensity of the solenoid) is:

$$
\begin{aligned}
& H_{0}=2 \pi n_{1} n_{2} I \times 0.035 \times \ln \frac{0.04+\left(0.04^{2}+0.035^{2}\right)^{1 / 2}}{0.022+\left(0.022^{2}+0.035^{2}\right)^{1 / 2}} \times 2 \\
& =0.17 n_{1} n_{2} I
\end{aligned}
$$

According to the relationship of coil turns $N$ of the solenoid and its center magnetic field intensity[8]:

$$
H=0.4 \pi \frac{N I}{l}=0.4 \frac{1500 I}{0.07}=26928 I(\mathrm{~A} / \mathrm{m})
$$

Because $H_{0}$ equals to $H$, we have:

$$
0.17 n_{1} n_{2} I=26928 I \Rightarrow n_{1} n_{2}=158400
$$

(7) is obtained by combining (6) with (3): 


$$
H_{x}=995256.54 I\left\{\begin{array}{l}
(x+0.035) \ln \frac{0.04+\left[0.04^{2}+(x+0.035)^{2}\right]^{1 / 2}}{0.022+\left[0.022^{2}+(x+0.035)^{2}\right]^{1 / 2}} \\
+(0.035-x) \ln \frac{0.04+\left[0.04^{2}+(0.035-x)^{2}\right]^{1 / 2}}{0.022+\left[0.022^{2}+(0.035-x)^{2}\right]^{1 / 2}}
\end{array}\right\}
$$

According to (7), the magnetic field intensity distribution of the solenoid is shown in fig.6:

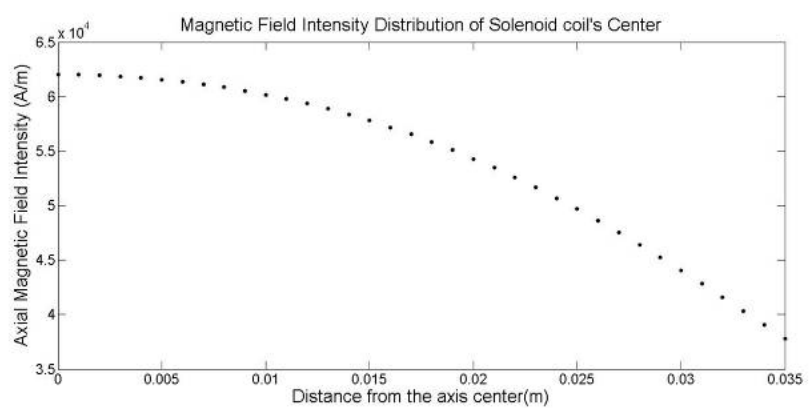

Fig.6 Magnetic field intensity distribution of solenoid coil's center

\section{Design of Micro-Driving System}

1) Structure of micro-driving system

The micro-driving system is consisted of micro-driving device based on GMM, magnetic field controlling system, measuring and controlling system of self-sensing micro-feed and so on (in fig.7)

\section{2) Process of measuring and controlling system for} micro-driving device

The micro-driving device based on GMM is controlled by computer (Fig.8). The control signal from the computer is to control the magnetic field of the GMM stick of the micro-driving device, and realize the needed micro feed output. The feedback controlling is realized by comparing the micro-feed output detected by the micro-displacement sensor.

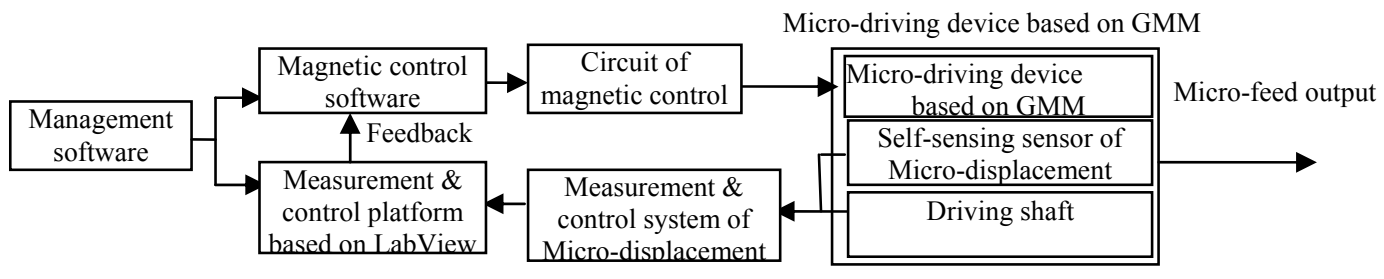

Fig.7. Micro-driving system based on GMM

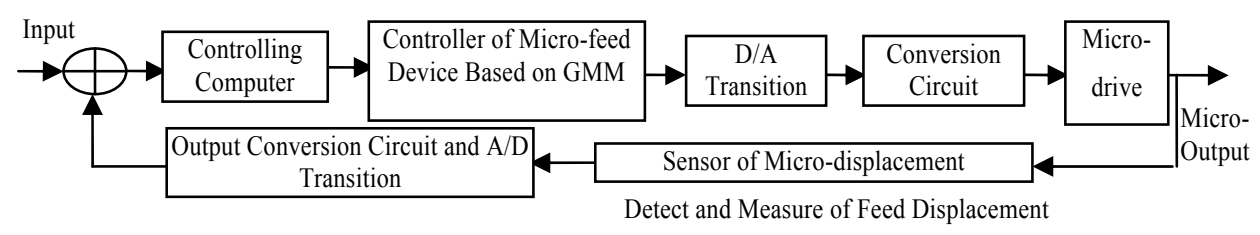

Fig.8. The control system of micro-driving device based on GMM

\section{3) Assembly design of micro-driving device}

Fig.9 is the assembly drawing of micro-driving device based on GMM, which shows that the micro-driving device[9] is assembled with a shell, a solenoid, a skeleton, a fore and back sealed cover, the GMM stick, an output shaft, the preloading spring, an adjusting screw nut and a cooling water pipe. When the solenoid is electrified, a magnetic field is set up, then the axial strain of the GMM stick is generated, and the axial feed of the shaft is generated. The cooling water flows through the cooling pipe to cool the GMM stick. According to the experiment, the accuracy of micro-feed of the micro-driving device is $0.1 \mu$, and its resolution is $0.01 \mu$.

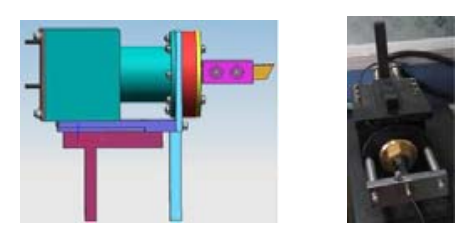

Fig.9. The assembly drawing of micro-driving device

\section{Research on Hysteresis Features of the Micro-Driving Device}

\section{1) Analysis of hysteresis loop of the micro-driving device}

When magnetic material is magnetized in the magnetic field, its magnetization intensity $M$ is not a signal value function of $H$, but depends on the history of magnetization. With the increasing of $H, M$ will be saturated. Even though $H$ is reduced to $0, M$ is not reduced to 0 with the change of $H$. As long as there is any residual magnetization $M_{r}$ existing, the change curve of $M$ is behind the curve of $H$ and deviated from the initial magnetization curve. The closed curve between $M$ and $H$ formed when the magnetic material is magnetized in a positive and negative cycle magnetic field is called hysteresis loop[10], seeing fig. 10: 


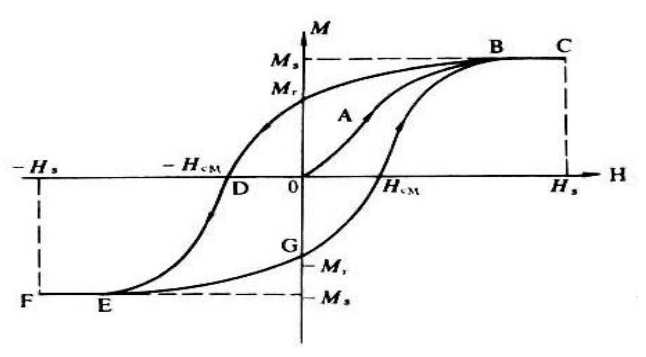

Fig.10. Hysteresis loop

According to the hysteresis loop of the micro-driving device based on GMM, its hysteresis loop is obtained by experiment between the micro-feed output and the current load on the solenoid. Under a given pre-pressure, the load current affected on the solenoid is $0-0.8 \mathrm{~A}$, $0-1.0 \mathrm{~A}, 1-1.2 \mathrm{~A}, 0-1.4 \mathrm{~A}, 0-1.6 \mathrm{~A}, 0-1.8 \mathrm{~A}$, and the changing steps is 40. Fig.11 shows the hysteresis loop of the micro-driving device under the pre-pressure 4.29MPa .

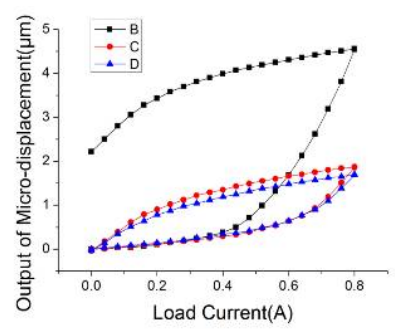

(a) Maximum load current $0.8 \mathrm{~A}$

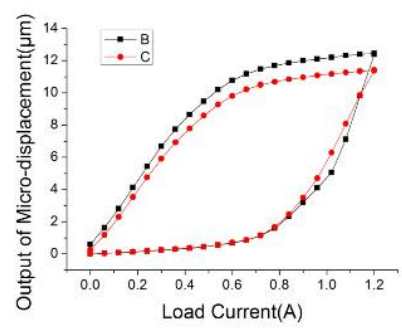

(c) Maximum load current 1.2A

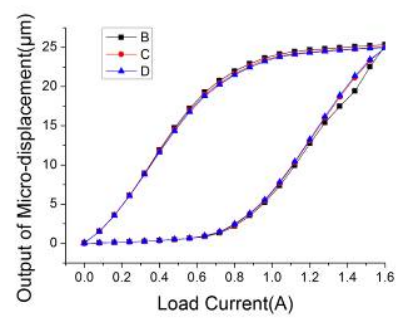

(d) Maximum load current $1.4 \mathrm{~A}$

(e) Maximum load current 1.6A (f) Maximum load current 1.8A Fig.11 Hysteresis loop of micro-driving device

In fig.11, B represents the first experimental curve, C represents the second experimental curve and $\mathrm{D}$ represents the third experimental curve. Fig.11 shows that, in a certain range, the larger the current is loaded, the better the loop coincidence degree of the micro-deriving device's micro-feed output is. The test is proved that when the load current is exceeded a certain value, its loop coincidence degree gets worse. Under the pre-pressure 4.29MPa, the load current for better loop coincidence degree is $1.6 \mathrm{~A}$ or $1.8 \mathrm{~A}$,and its magnetic field intensity is $H_{1.6}=43084.8 \mathrm{~A} / \mathrm{m}$ and $H_{1.8}=48470.4 \mathrm{~A} / \mathrm{m}$.

\section{2) Sluggish}

The sluggish of magnetic material is defined as[11]:

$$
f=\frac{\max \left(X_{2 i}-X_{1 i}\right)}{Y_{F S}} \times 100 \%
$$

$\operatorname{In}(8), X_{1 i}$ and $X_{2 i}$ indicate the feed output of the micro-deriving device under the same current while loading and unloading respectively, $Y_{F S}$ is output range.

According to fig.11(e), the sluggish curve of the micro-driving device is shown in fig.12 (load current is 1.6A):

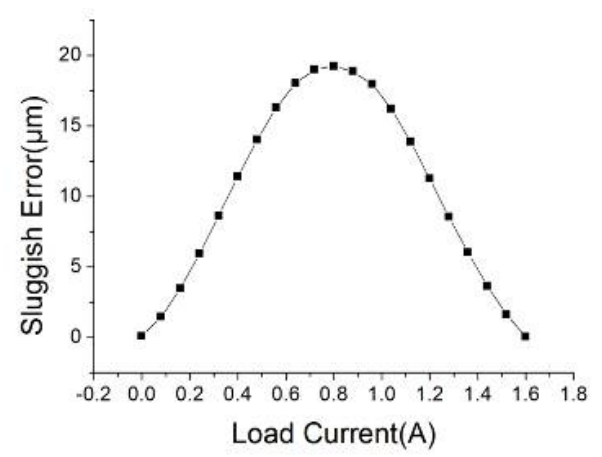

Fig.12. Sluggish curve of the micro-driving device under load current $0-1.6 \mathrm{~A}$

From fig.12, the sluggish of the micro-feed output increases at first, then decreases. The load current is $0.8 \mathrm{~A}$ at the point of the largest sluggish, whose value is $77.1 \%$. Fig. 13 shows the largest sluggish error under different load currents.

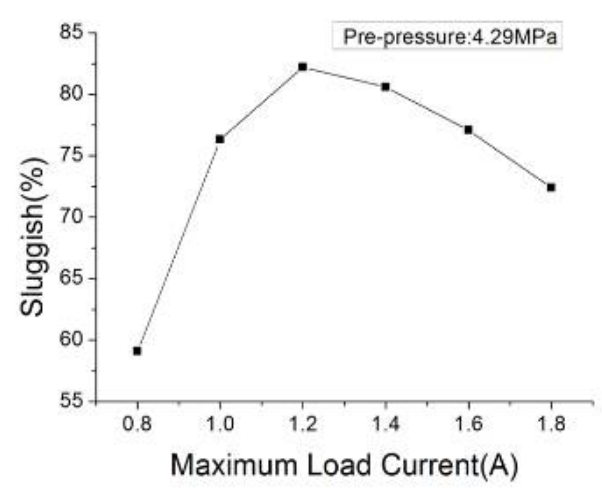

Fig.13. The largest sluggish error under different load current

In fig.13, the sluggish increases at first, then decreases, and there is a certain load current $(1.2 \mathrm{~A})$ making the largest sluggish error, hereby the best current load on the micro-driving device is larger than $1.2 \mathrm{~A}$.

\section{Research of MeAsuring and Detecting System OF MICRO-FEED SELF-SENSING}

\section{A. The Principle of Micro-Feed Self-Sensing}

The principle of micro-feed self-sensing is that the micro-feed of the micro-driving device is real-time measured using micro-feed sensor. Material such as $35 \mathrm{CrNiMoA}[12]$ is used to make elastic thin plate as a 
elastic sensitive element, and high accuracy resistance strain gauge such as BF350-3AA is used too. Drawing of the micro-feed self-sensing sensor is seen in fig.14. When its right face is blocked by a baffle, the thin plate is deformed because of the micro feed produced by the driving of the GMM stick. According to the relationship between micro-strain of the elastic thin plate and the output of the sensor, the function of micro-feed self-sensing is realized.

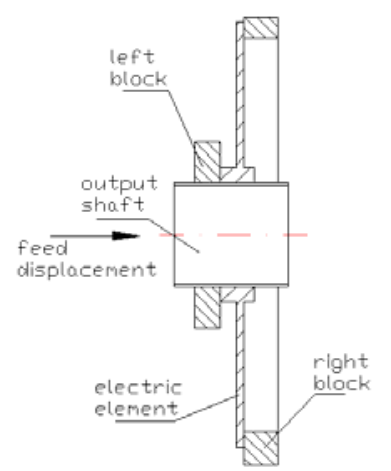

Fig.14. Structure of micro-feed self-sensing sensor

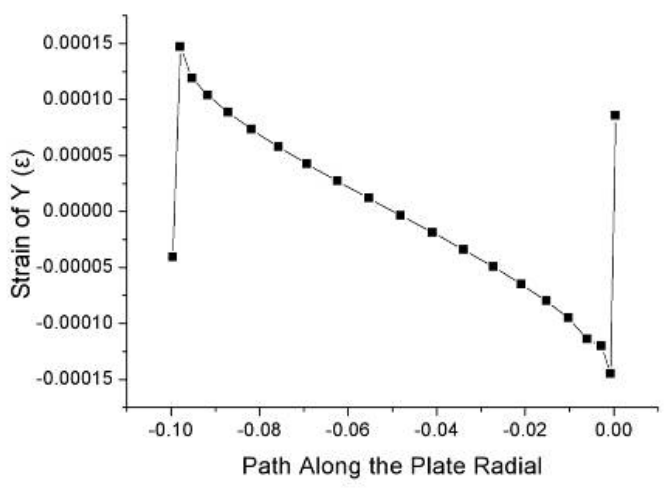

Fig.15. Micro-strain output along the plate radial path

Fig.15 is its relationship between the displacement and the strain of a path from left to right through the thin plate center face, where the horizontal coordinate indicates distance of the path and the vertical coordinate indicates the strain. FEM analysis demonstrates that the micro strain and the output feed of the device have a linear relationship, and its micro-feed value can be measured by the sensor, the largest strain being $15 \mu \varepsilon$ located on the edge and in the center of the plate.

The resistance strain gauge[13-17] is a sensor whose resistance would change with the strain. In general, the metal resistance strain gauge is selected for high temperature stability, good linearity and reliability, in order to enlarge sensitivity and reduce measurement error influenced by the temperature, a full bridge circuit of the resistance strain gauge is connected (seeing fig.16), for some advantage of the resistance strain gauge, which can achieve micro-feed self-sensing function of the micro-driving device. The resistance of the metal wire is calculated by (9):

$$
\begin{aligned}
& d R=d \rho \frac{l_{\text {wire }}}{S}+d l_{\text {wire }} \frac{\rho}{S}-d S \frac{\rho l_{\text {wire }}}{S^{2}} \\
& \Rightarrow \frac{d R}{R}=\frac{d \rho}{\rho}+\frac{d l_{\text {wire }}}{l_{\text {wire }}}-2 \frac{d r}{r}
\end{aligned}
$$

In (9)and(10), $R$ is the resistance, $\rho$ is the resistivity of the metal wire, $l_{\text {wire }}$ is the length of metal wire, $S$ is the cross-sectional area of metal wire, and $r$ is the radius of metal resistance wire. Where $\varepsilon_{l}=d l_{\text {wire }} / l_{\text {wire }}$ (axial strain) and $\varepsilon_{r}=d r / r$ (radial strain), $\varepsilon_{r}=-\mu \varepsilon_{l}$ (where $\mu$ is poisson's ratio of metal resistance wire), if the metal wire is loaded by pull force, it would be lengthen along axial direction and shorten along radial direction, thus (10) is gotten:

$$
\begin{aligned}
& \frac{d R}{R}=\frac{d \rho}{\rho}+\varepsilon_{l}-2 \varepsilon_{r}=\frac{d \rho}{\rho}+(1+2 \mu) \varepsilon_{l} \\
& \Rightarrow \frac{d R / R}{\varepsilon_{l}}=(1+2 \mu)+\frac{d \rho / \rho}{\varepsilon_{l}}=K_{l}
\end{aligned}
$$

$\operatorname{In}(10): K_{l}$ is strain sensitivity coefficient of metal resistance wire. The value of strain is derived from the resistance's change value of the bridge circuit (see fig.16).

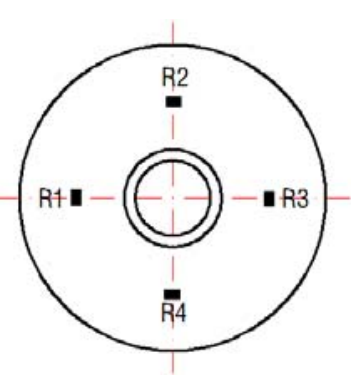

(a)

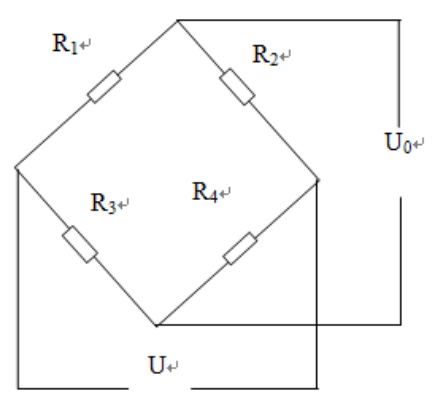

(b)
Fig.16. Installation of resistance strain gauge and its full bridge circuit

The differential full bridge circuit connection of resistance strain sensor is shown in fig.18. As to balance the circuit, we have $R_{1}=R_{2}=R_{3}=R_{4}=R$, and to reduce the influence of the temperature and ignore the error caused by temperature, the effect of horizontal strain of the gauge was taken into consideration[11]. In that case, as is seen from fig.16(b), the relationship between the output voltage $\left(U_{0}\right)$ of the bridge and the input voltage $(U)$ is:

$$
U_{0}=\frac{2 n l+(n-1)(1-\mu) \pi r_{\text {shan }}}{2 L} K_{l} \varepsilon_{l} U
$$

where $n$ denotes the wire number of the strain gauge, $L$ denotes the length of the strain gauge, and $r_{\text {shan }}$ denotes the radius of the strain grid. According to (11), the micro feed is gotten from the strain of resistance strain gauge, which is gotten from the output voltage $\left(U_{0}\right)$ of the measurement bridge circuit.

\section{B. Production of Micro-Feed Self-Sensing Sensor with Precision Strain Gauge}

The precision strain gauge such as BHF350-2AA is selected to fabricate the micro-feed self-sensing sensor of the micro-driving device.

The process of production includes three steps, first, cleaning the position of the elastic element where the strain 
gauge would be pasted on; second, pasting the strain gauges on the position with high performance paste glue (type is HH-2000-610), pressuring and solidifying them; at last, welding electric bridge with wire. Fig.17 shows the sensor where the strain gauge is pasted, which has been tested with the resistance value between adjacent pins of the full bridge being $263 \Omega$ and that between diagonal pins being $351 \Omega$.

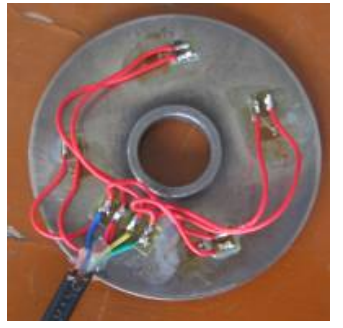

Fig.17. Sensor pasted the strain gauge

\section{Calibration of Micro-Feed Sensor}

When the micro-feed self-sensing sensor is finished, the sensor need to be calibrated dynamically and statically to confirm the relationship between micro-feed input and micro strain output, and calibration consists of two steps.

1) To calibrate the sensor between force input and micro strain output

The methods to calibrate the sensor between force input and micro strain output is to test the micro strain output of the sensor when the force load is loaded with some standard weight on the elastic plate where its loaded diameter is $\phi 79.74 \mathrm{~mm}$ and its loaded ring width is $1 \mathrm{~mm}$ (seeing fig.18), then the experimental data is analyzed by the least squares linear fit, and the least squares linear fit curve is seen in fig. 19.

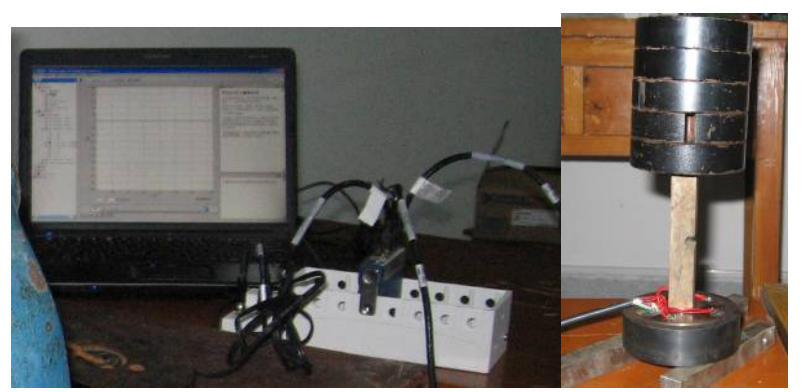

Fig.18. To calibrate the sensor between force input and micro strain output

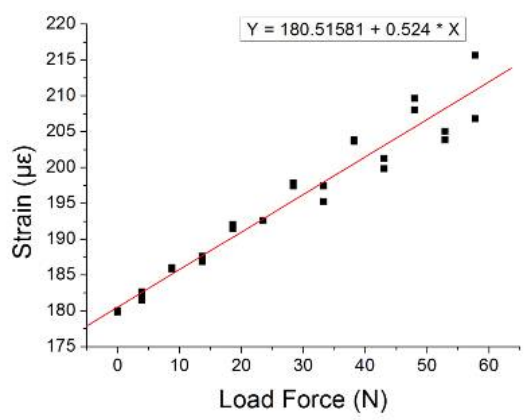

Fig.19. The least squares linear fit curve between force input and micro strain output

The linear function expression (12) between force input and micro strain output of the sensor is concluded according to fig. 20 .

$$
Y=0.524 X+180.51581
$$

The standard deviation of the least squares linear fit is $2.33042 \mu \varepsilon$.

2) To calibrate the sensor between micro-feed input and micro strain output

The calibration between micro feed input and micro strain output follows behind the calibration between force input and micro strain output. During the calibration, the sensor of micro-feed self-sensing and the LVDT micrometer are used. The calibration method is to change the input current loaded on the micro-driving device, then the micro strain output is achieved, and the micro feed of the output shaft is tested from the two sensor respectively (seeing fig.20)(one is the micro-feed self-sensing sensor and the other is the standard micro-displacement sensor named as Micrometer). Eventually, the calibration curve could be drawn by analyzing the experiment data (shown in fig.21).

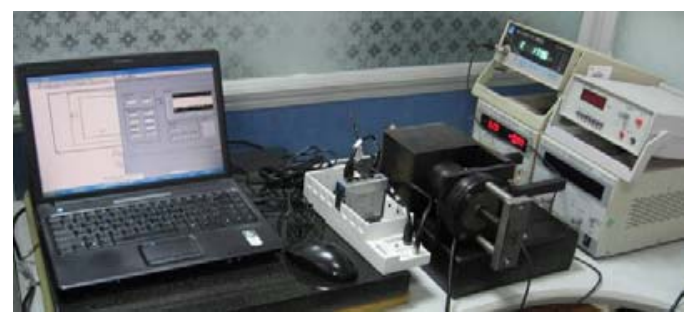

Fig. 20. Calibration between micro feed input and micro strain output

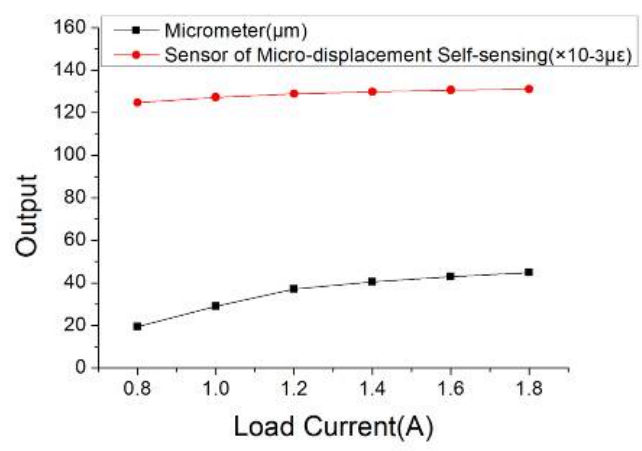

Fig. 21. The output relationship between micrometer and micro-feed self-sensing sensor

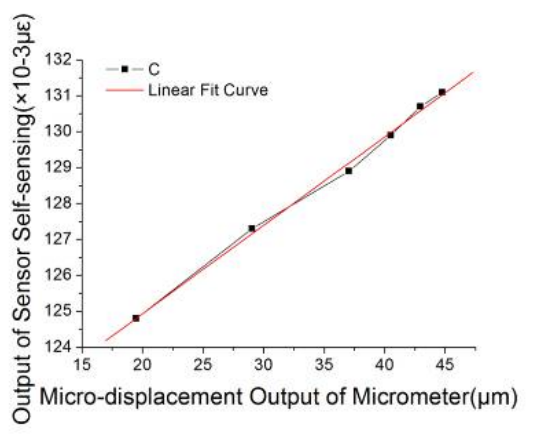

Fig. 22. Calibration between micro strain and micro feed

Fig.22 shows the relationship curve between micro strain and micro feed of the micro-driving device, which is linear. The least squares linear fit function between micro strain and micro feed of the device can be expressed by (13):

$$
y=120.01271+0.24609 x
$$


In(13), $y$ denotes the micro strain output of the micro-feed self-sensing sensor $\left(\times 10^{-3} \mu \varepsilon\right), \quad x$ denotes the micro feed input of micrometer $(\mu \mathrm{m})$. The largest fit nonlinear error $\Delta_{\max }$ is $0.15545 \times 10^{-3} \mu \varepsilon$, and according to fig.23, $y_{F S}$ is $6 \times 10^{-3} \mu \varepsilon$, hereby the fit linearity $e_{1}$ is:

$$
e_{1}=\left|\Delta_{\max } / y_{F S}\right| \times 100 \%=0.15545 / 6 \times 100 \%=2.6 \%
$$

Formula (15) is derived from (13):

$$
x=4.04968 y-485.89205
$$

According to (15), when the micro-driving system is in the magnetic field, the micro strain $(y)$ of the micro-feed self-sensing is tested, then the micro feed of the micro-driving device $(X)$ is calculated by (15), thus the function of the micro-feed self-sensing of the device is realized, and its sensitivity $S_{n}$ is gotten as:

$$
S_{n}=d x / d y=4.04968
$$

\section{EXPERIMENT RESEARCH OF MICRO-FEED SELF-SENSING FunCTION FOR THE MiCRO-Driving DEVICE BASED ON GMM}

\section{A. Experiment Equipment Needed}

After finishing the calibration of the sensor of micro-feed self-sensing, the experiment of micro feed output of the micro-driving device is needed, with equipments include micro-driving device based on GMM, controlling and driving system of magnetic field for GMM, constant voltage power supply, NI's dynamic strain measurement system, data acquisition card and computer.

\section{B. Result Analysis of the Experiment}

By controlling the micro-driving device with stable current and dynamic current, the micro output tested by the micro-feed self-sensing sensor is gotten.

\section{1) Stable micro-feed output experiment of}

micro-driving system

The micro-driving system is controlled by stable current such as $0.8 \mathrm{~A}, 1.0 \mathrm{~A}, 1.2 \mathrm{~A}, 1.4 \mathrm{~A}, 1.6 \mathrm{~A}, 1.8 \mathrm{~A}$, and the micro-feed output is tested by micro-feed self-sensing sensor (seen in fig.23).

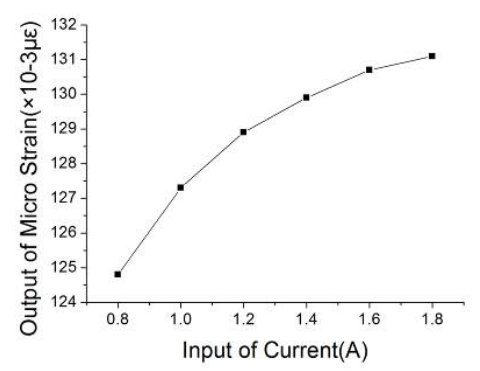

Fig.23. Relationship between micro strain output of micro-driving system and input stable current

In fig.23, the input stable current is $0.8 \mathrm{~A}, 1.0 \mathrm{~A}, 1.2 \mathrm{~A}$, $1.4 \mathrm{~A}, 1.6 \mathrm{~A}$ and $1.8 \mathrm{~A}$, and the output value is the average micro strain of the micro-driving system. According to fig.23, the relationship between input stable current and output micro strain is a rising nonlinear curve, of which the nonlinear feature is related with the nonlinear feature of the GMM.

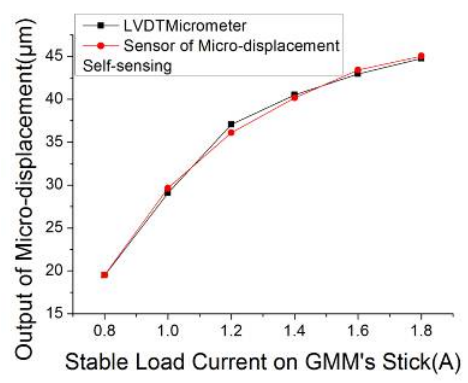

Fig.24. Output contrast between micrometer and micro-feed self-sensing sensor under stable current input

Fig.24 shows that the output of the sensor of micro-feed self-sensing is in coincidence with that of micrometer.

2) Dynamic experiment research of micro-driving system

The load dynamic current is changing from 0 to the maximum value and then unload from maximum value to 0 , with each variation value of $I_{\max } / 20$. The output is tested by micrometer and micro-feed self-sensing sensor separately (seeing fig.25 and fig.26).

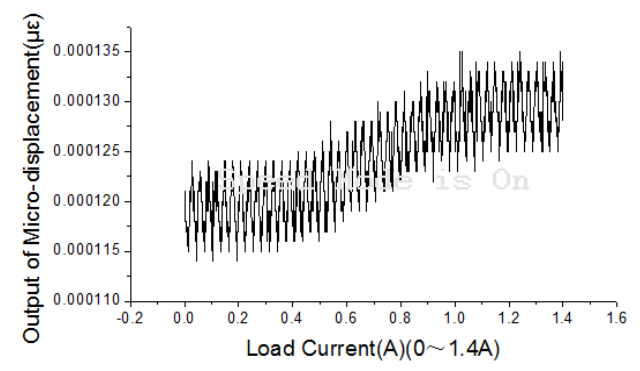

Fig.25. Micro strain output of micro-feed self-sensing

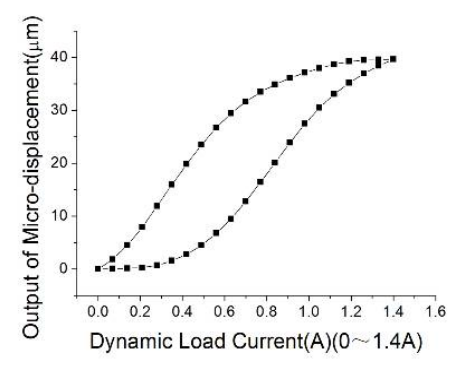

Fig.26 Micro feed output of micrometer

Fig.25 shows the micro strain output of the micro-driving device tested by micro-feed self-sensing sensor, illustrating that the sensor can reflect the real-time dynamic output of the micro-driving device. Fig.26 shows that the micrometer only reflects the stable output of the device, if the real-time dynamic output is needed, the micro-feed self-sensing sensor is essential.

\section{CONCLUSIONS}

Research shows that the micro-driving system based on GMM can be realized submicron feed output and the function of micro-feed self-sensing, which can be applied in the field of super precision manufacturing or other situations needing micro feed. Comparing with the existing 
micro-driving devices, the micro-driving system based on GMM has some advantages as follows: first, the submicron feed could come true with convenient control; second, feed feedback control is accomplished by the feed self-sensing sensor fixed on the micro-feed device.

Overall, the application of the micro-driving system based on the GMM has a bright future.

\section{ACKNOWLEDGEMENT}

The research is supported by doctoral fund of Ministry of Education (20090143110005) and the Opening Project of Guangxi Manufacturing Systems and Advanced Manufacturing Technology Laboratory (08037).

\section{REFERENCES}

[1] WANG Bo-wen,"The Design of Device and Preparation of GMM,"BeiJing: Metallurgy Industry Press,2001(in Chinese).

[2] Fennimore A M,Yuzvinsky T D, Han W Q, et al,"Rotational actuators based on carbon nanotubes,"Nature, Vol.424, No.6947, pp.408-410,2003.

[3] JIA Zhen-yuan, YANG Xing, WU Dan,et al.,"Application in the Field of Fluid Control Components with Magnetostrictive Actuator,"Machine Tool \& Hydraulics, pp.1-4,2,2000(in Chinese)

[4] Ai-hua MENG, Ming-fan LI, Yu-liang PAN, et al.,"Performance of Pulsed Jet on-off Valve Based on Giant Magnetostrictive Actuator, "Transactions of the Chinese Society for Agricultural Machinery, Vol. 41, No.8,pp.211-215,2010(in Cinese).

[5] XU Ai-qun,"Method of Automatic Following Resonance Frequency for Giant Magnetostrictive Transducer, "Proceedings of the CSEE, Jul. 25,Vol.29,No.21,pp.114-118,2009

[6] XU Feng, LI Qing-xiang,"Precision Design of Machinery".BeiJing,2005(in Chinese).

[7] LU Quan-guo,"Research and Application of Micro-actuator Based on GMM, "Wuhan: Wuhan University of Technology,2007(in Chinese).

[8] LIN Qi-ren, ZHAO You-min,"Principles of Magnetic Circuit Design".BeiJing,1987(in Chinese).

[9] CHEN Ding-fang, LU Quan-guo, LI Li-fang,et al.,"Matro-actuator of Super Precision Based on GMM, "China Patent:ZL200520099220.0,2007(in Chinese).

[10] Potersenke: Silicon as a mechanical. C. Proc IEEE, 70, 1982.

[11] LIU Ying-chun, YE Xiang-bin,"The principle, design and application of sensor,"Chang Sha,2006(in Chinese).

[12] WANG Qi-yi,"China mechanical Design Ceremony,"Vol. 2, NanChang, 2002(in Chinese).
[13] LI Juan, XU Hong-hai, YUAN Hai-qiang,"Trend Analysis of Cutting Force Measurement," Modern Busimess Industry, Vol.19, No.6, pp.180-181, 2007(in Chinese).

[14] YANG Zhao-jian, WANG Qin-xian,"Research Development of Force Sensor," ShanXi Science and Technology, pp.5-9,2003(in Chinese).

[15] WANG De-jie,"Application of Measurement System Used Strain Gauge on NC tools,"Heavy Machinery and Technology,12(4),pp. 30-32,2006(in Chinese).

[16] MA Bin-hui,LU Ze-sheng,"Research of Small Micro-force Sensor,"Chinese Journal of Mechanical Engineering (Supplement),5,pp. 227-230,2006(in Chinese).

[17] YU Zhen, CHEN Ding-fang, YANG Yan-fang, et al.,"Research of Displacement Self-apperceive Micro-feed Tool Carrier Based on GMM,'ISA 2010, Vol.1, Wuhan, China, May 22-23, pp.190-193, 2010

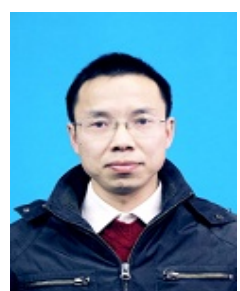

YU Zhen was born in AnHui Province, China in 1973, and received master of engineering in materials from HuaZhong University of Science and Technology,WuHan,China,in 2003.

From 2007 to 2010, has been received Ph.D. degree in mechanical manufacturing and automation at WuHan University of Technology,WuHan,China, and now he is a lecturer and a research fellow at the School of Machinery \& Automation, WuHan University of Science and Technology, China. His main research interest is precision manufacturing technology and theory.

$\mathrm{He}$ is a senior member of IACSIT.

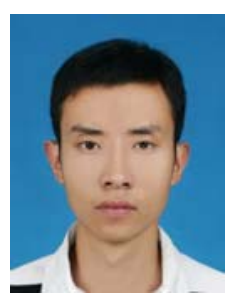

MEI Jie was born in HuBei Province, China in $1985 . \mathrm{He}$ is currently a Ph.D. candidate in mechanical manufacturing and automation at Wuhan University of Technology. He is doing some researches on Virtual Reality, diagnosis, precision manufacturing and so on, as well as publishing more papers, most of which were indexed by EI and ISTP.

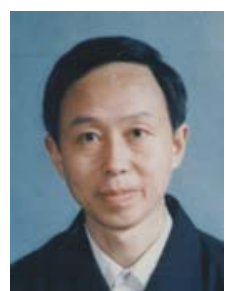

Chen Ding-fang was born in HuBei Province,China in 1946 and he is a Professor and National experts of Wuhan University of Technology.He is a specialist in advanced manufacturing and intelligent manufacturing.He also works in the area of MEMS and complex system and optimization.He has been published more than two hundred papers most of which were indexed by EI and IST. 